\title{
Thermal excitation of large charge offsets in a single-Cooper-pair transistor
}

\author{
L.R.Simkins ${ }^{1}$, D.G.Rees ${ }^{1, \text { a) }}$, P.H.Glasson ${ }^{1}$, V.Antonov ${ }^{1}$, E.Collin ${ }^{2}$, P.G.Frayne ${ }^{1}$, \\ P.J.Meeson ${ }^{1}$, M.J.Lea ${ }^{1, b)}$ \\ ${ }^{1}$ Department of Physics, Royal Holloway, University of London, Egham, Surrey, \\ TW20 OEX, United Kingdom. \\ ${ }^{2}$ Institut Néel, CNRS \& Université J. Fourier, 25 rue des Martyrs, BP166, F-38042 \\ Grenoble Cedex 9, France.
}

18 June 2009

Charge offsets and two-level fluctuators are common in single-electron transistors (SET) with a typical magnitude $|\Delta Q|<0.1 e$. We now present measurements in a $2 e$-periodic single-Cooper-pair transistor (SCPT) which exhibited hysteretic charge offsets close to 1e. The real-time capture and escape of individual electrons in metastable trapped states was measured at very low temperatures. This enabled the dynamics of the transitions to be investigated in detail, demonstrating thermal excitation to a hysteretic tunneling transition. We also show that, allowing for the hysteresis, the metastable states are in thermal equilibrium with each other. The observed temperature dependence and hysteresis can be explained by the coupling of a two-level fluctuator to a quasiparticle trap.

PACS numbers: 73.23.Hk, 72.20.Jv, 73.40.Rw

Corresponding author: M.J.Lea, m.lea@rhul.ac.uk

\footnotetext{
a) Present address: Low Temperature Physics Laboratory, Riken, Hirosawa 2-1, Wako-shi, 351-0198 Japan.

b) Author to whom correspondence should be addressed. Electronic mail:m.lea@rhul.ac.uk.
} 


\section{INTRODUCTION}

The detection and control of localised electrons in both intrinsic and fabricated traps in solid-state devices are major technical challenges. Single-electron transistors (SET) and single-Cooper-pair transistors (SCPT) are used as sensitive electrometers in charge qubits ${ }^{1,2}$, quantum $\operatorname{dots}^{3}$ and Cooper-pair boxes ${ }^{4}$. But these devices exhibit intrinsic charge traps and two-level systems ${ }^{5,6,7,8}$, giving charge offsets, two-level fluctuators, hysteresis and $1 / f$ noise ${ }^{9}$, which limit their performance. It is clear that individual microscopic two-level fluctuators (TLF) play an important role, in which trapped charge jumps between two distinct and physically separated sites. These can result in conductance fluctuations and in background charge noise. Each fluctuation corresponds to the capture or escape of a trapped electron, from an atomic defect or a small conducting island. The trapped electron can come from another electron trap or from conducting electrodes in the device, by thermal activation or tunneling. Many experiments have tried to elucidate the nature, and location, of these defects. An understanding of these defects is crucial if the full range of potential technical applications for charge sensitive detectors is to be realised.

We now present a series of new experiments which investigated the detailed dynamics of some charge offsets in SCPT. Individual electron transitions were detected and measured. A novel feature of this work was the use of both $1 e$ and $2 e$ periodic Coulomb blockade oscillations $(\mathrm{CBO})$ which revealed charge offsets close to $1 e$ in magnitude. A preliminary account of this work has been published ${ }^{10}$.

A brief summary of previous relevant work is given here. Ralls et al. ${ }^{11}$ studied resistance switching in Si inversion layers due to the thermally activated capture and escape of electrons from the inversion layer into traps at the $\mathrm{Si} / \mathrm{SiO}_{2}$ interface above $4 \mathrm{~K}$. This gave telegraph noise where the On and Off times, $\tau_{1}$ and $\tau_{2}$, are the times to escape from and be captured by a trap. As a gate voltage was swept, the capture and escape rates were given by

$$
1 / \tau_{i}(V, T)=\left(1 / \tau_{0 i}\right) \exp ( \pm e V / \gamma k T)
$$

where the $+\mathrm{ve}$ sign corresponds to electron capture and the -ve sign to electron escape and $1 / \gamma(\gamma$ was typically 5$)$ corresponds to the coupling strength of the electrode to each TLF. The prefactors $1 / \tau_{0 i}$ were thermally activated $\propto \exp \left(-E_{\mathrm{a}} / k T\right)$ with a range of 
activation energies $E_{\mathrm{a}}$. Rogers and Buhrman $^{9}$ studied the noise in conductance fluctuations in small tunnel junctions from individual Lorentzian fluctuators. These experiments showed the importance of the details of the dynamics of individual defects and how a broad range of attempt frequencies and activation energies leads to $1 / f$ noise in tunnel junctions.

Cobden, Uren and Pepper ${ }^{12}$ found two types of TLFs in MOS FETs. The first type corresponded to tunnelling from an inversion layer to a near-interface oxide defect, while the second was a two-state system weakly coupled to an electron bath. In both cases the ratio of the relaxation times was given by

$$
\tau_{1} / \tau_{2}=\exp \left(e\left(V-V_{0}\right) / \gamma k T\right)
$$

but the geometric mean switching rate $\left(\tau_{1} \tau_{2}\right)^{-1 / 2}$ of the second type was a narrow Lorenztian peaked at the resonance voltage $V_{0}$, whose width depended on the dissipative coupling strength to the electron bath, as given by Grabert and Weiss ${ }^{13}$.

Similar fluctuators produce background charge noise in Coulomb blockade electrometers, or single-electron transistors (SET). The moving charge changes the polarisation of the SET island which leads to random telegraph noise in the source-drain current $I_{\mathrm{SD}}$, with characteristic switching times which can be as long as a second ${ }^{14}$. Zorin et al. ${ }^{15}$ measured this background charge noise in a coupled pair of $\mathrm{Al}-\mathrm{AlO}_{x}$-Al SETs using dual channel analysis, though individual fluctuators were not observed. Because of the high correlation value, $0.15 \pm 0.05$, between the $1 / f$ noise in both SETs, they concluded that the noise sources were in the $\mathrm{Al}_{2} \mathrm{O}_{3}$ substrate. Tavkhelidze and Mygind ${ }^{16}$ showed that low frequency noise could be frozen out by slow thermal annealing to below $100 \mathrm{mK}$, and was strongly affected by microwave irradiation.

A systematic study of TLFs was made by Zimmerman et al. ${ }^{17}$, who unambiguously demonstrated that they were not in the tunnel barriers. They found that a cluster of TLFs, with coherent changes in charge configurations (in which a single TLF event triggers others), leads to multi-level cascades of charge shifts. Individual TLF switches were typically of magnitude 0.1 to $0.2 e$, while the cluster size may be as large as 50 defects.

Krupenin et al. ${ }^{18}$ fabricated an Al SET directly on the oxidised base electrode to separate conductance fluctuations from the background charge noise on the substrate. 
They concluded that conductance fluctuations and background charge noise were uncorrelated, though both came from fluctuators.

However, Kenyon et al. ${ }^{19}$ studied one TLF in the tunnel barrier in an SSET and SET. In this case the transition rates $\tau_{1}$ and $\tau_{2}$ depended periodically on the Coulomb blockade oscillations as the gate voltage $V_{g}$ was swept. This was interpreted as due to the inelastic scattering of tunneling electrons from the charged fluctuator. At low temperatures, the rates became independent of temperature due to direct quantum tunneling between the states.

Fujisawa and Hirayama ${ }^{20}$ used an rf-SET to study high-frequency charge noise in a quantum dot and, in particular, telegraph noise from a nearby electron trap. The escape and capture times of the trap depended on gate voltage and were characteristic of a Poisson process with independent capture and escape. Good agreement was found with a model of electron tunneling from the metallic leads with $\tau_{0}=12 \mu \mathrm{s}$, and an effective temperature $T_{\text {eff }}=5.3 \mathrm{~K}$, corresponding to $\gamma T$ in Eq.(2).

The prospect of Quantum Computing and Quantum Information Processing gave a new impetus to studies of SET charge noise, particularly for the long-term stability. Further investigations ${ }^{21}$ showed that the long-term drift, which can give cumulative charge offsets $\Delta Q>1 e$ in Coulomb blockade devices, is not attributable to extrapolation of the $1 / f$ noise and is driven by a different mechanism. Changes in fabrication seemed to make little difference. But the characteristic rate at which a given SET exceeded a pre-set charge offset decreased as $1 / t$ for several weeks. It was suggested that the slow relaxation of intrinsic TLFs in the substrate could explain this annealing. More promisingly, it was found that the long-term drift in Si-based SETs was very much less than in metal devices $^{22,23}$.

Grupp et al. ${ }^{24}$ studied the anomalous transport through an SET, due to coupling between the SET island (made of bismuth) and an intrinsic TLF. They showed that the TLF state, and hence the offset charge, was determined dynamically and self-consistently by electrostatic coupling as electrons enter the island. They introduced a TLF transition rate $\Gamma_{\mathrm{TLF}}$ which was constant if the energy is lowered during the switching and $\Gamma_{\mathrm{TLF}} \exp (-\Delta E / k T)$ if the overall energy was raised by $\Delta E$. If $\Gamma_{\mathrm{TLF}}>\mathrm{SET}$ tunnelling rate, 
then each electron in the source-drain current through the island switched the TLF. Otherwise, the TLF would switch telegraphically.

Furlan and Lotkhov ${ }^{6}$ used an SET with multiple gates to study the static charge offset problem in a normal state Al SET at $5 \mathrm{mK}$, though the electrons were at $45 \mathrm{mK}$. Reproducible non-zero charge offsets $\Delta Q$ (typically $<0.1$ e) occurred at specific gate voltages, with about 20 transitions/volt swept. A key feature was that most charge shifts demonstrated hysteresis in the gate voltage, with a wide range of different magnitudes, corresponding to bistable traps close to the SET island. Individual traps could be characterised by the charge offset, the hysteresis and the dependence on different gate voltages. The use of multiple gates enabled the position of the traps to be determined, which were all outside the tunnel barriers, and within a few $\mathrm{nm}$ of the SET island. Interactions between traps were observed. Test experiments in the SET superconducting state gave identical results. The dynamics could not be measured as all the transition rates were faster than the measurement bandwidth $(\sim 10 \mathrm{kHz})$. The origin of the traps and hysteresis could not be determined, but this gave an excellent account of the experimental features of TLFs in an SET.

Buehler et al. ${ }^{25}$ studied MOS devices with a high-bandwidth (10 MHz) rf-SET to measure telegraph noise with switching times in the sub- $\mu$ s range. They found telegraph noise on all time scales with $\Delta Q<0.1 e$, without hysteresis. The traps were located in the interface regions between the amorphous oxide and the $\mathrm{Si}$ or $\mathrm{Al}$, with an interface trap density $10^{12} \mathrm{~cm}^{-2}$. No change in trap behaviour was seen up to $500 \mathrm{mK}$, suggesting tunnelling between two trapping states. No voltage dependent relaxation rates were presented.

A detailed study was presented by Brown, Sun and Kane ${ }^{7}$ for Al SETs on an oxidised silicon substrate. TLFs were found in all their SET devices. They showed that the traps must be above the substrate surface and suggested that small isolated Al grains near the SET island might be responsible.

Zimmerman et $a l{ }^{26}$ presented a compendium of many measurements of charge offset drift in both Al-based and Si-based SETs. They show that the drift in Si-based SETs is consistently very much less, often at the level of $0.01 e$ over long periods. TLFs are found in Si devices, but are much more stable and reproducible. Metal SETs can be 
stable for many days, as in our devices, but are still subject to random shifts. They presented a model of non-equilibrium relaxation of charged defects, with a distribution of TLFs. They conclude that interactions between TLFs in metal SETs lead to the long-term drift, while in Si devices the interactions are mimimal. They speculated on possible material-specific causes of the drift: (i) hydroxyl ions, $\mathrm{OH}^{-}$, in the $\mathrm{AlO}_{x}$ or on the surface (ii) the density of $\mathrm{Al}$ in $\mathrm{AlO}_{x}$ will vary in a stoichiometry gradient, not in thermodynamic equilibrium, (iii) condensed Al metallic droplets and (iv) film mechanical stress.

Trapped electrons can escape from a metastable quantum state by tunneling ${ }^{27}$ through a potential barrier or thermal activation over the barrier at a temperature $T$. The escape rate is then proportional to $\exp (-E / k T)$ with an activation energy $E$. In some systems, the potential barrier can be driven to zero ${ }^{28}$ by an external control parameter $v$ with $E \propto v^{\xi}$ and $\xi \geq 1$. Trapped electrons can also escape by phonon-assisted tunneling, or tunneling from thermally excited energy levels ${ }^{29}$.

We now present new measurements of the detailed dynamics of the capture and escape of individual electrons in metastable trapped states at very low temperatures in an SCPT.

\section{DEVICE FABRICATION AND OPERATION}

The devices, designed for applications in surface-state charge detection ${ }^{30}$ using an $\mathrm{Al}-\mathrm{AlO}_{x}-\mathrm{Al} \mathrm{SET}^{31}$, were fabricated with multiple metallic layers using electron-beam lithography. An SEM image of one device is shown in Fig. 1. The substrate was a standard $\mathrm{Si}$ wafer covered with $200 \mathrm{~nm}$ of thermal oxide $\mathrm{SiO}_{2}$. The first, or lower, layer for electrical leads and gate electrodes was thermally evaporated $50 \mathrm{~nm}$ gold ( $\mathrm{Au}$ ) with a $\mathrm{Nb}-\mathrm{Cr}$ flash for adhesion. A channel structure for liquid helium was fabricated with 480 $\mathrm{nm}$ of thermal $\mathrm{SiO}_{2}$ covered with an upper guard layer of $80 \mathrm{~nm}$ gold. In the final stage, the SET was fabricated within the channel using standard shadow evaporation techniques. Two thermal evaporations of $35 \mathrm{~nm}$ of $\mathrm{Al}$ were made at different angles for the SET source and drain (which overlapped the gold lead electrodes) and for the SET island. Oxidation between the two $\mathrm{Al}$ evaporations provides the two tunnel barriers to the SET island. Full details of the fabrication procedures are given elsewhere ${ }^{32}$.

Voltages were applied through low pass filters and a $1 \mathrm{~m}$ length of thermocoax to drive the SET and to bias a set of gate electrodes. The source-drain current $I_{\mathrm{SD}}$, measured 
with a DL1211 current preamplifier, exhibited a Josephson-quasiparticle tunneling (JQP) peak and a dissipative Josephson branch, Fig. 2. Typical SET parameters were normal state resistance $28 \mathrm{k} \Omega$, Coulomb energy $54 \mu \mathrm{eV}$, Josephson energy $20 \mu \mathrm{eV}$ and superconducting energy gap $2 \Delta=360 \mu \mathrm{eV}$.

Coulomb blockade oscillations (CBO) were observed in $I_{\mathrm{SD}}$ when sweeping a gate voltage $V_{\mathrm{G}}$, due to capacitive coupling $C_{\mathrm{G}}$ to the SET island inducing a charge $Q=$ $-C_{\mathrm{G}} V_{\mathrm{G}}$. On the JQP peak, the $\mathrm{CBO}$ were periodic in $Q=1 e$, Fig. 3(a). At small sourcedrain voltages $\left(V_{\mathrm{SD}}<25 \mu \mathrm{V}\right)$, large amplitude $\mathrm{CBO}(>400 \mathrm{pA})$ were $2 e$ periodic $^{33}$, Fig. 3(b), when the superconducting island was in an even-parity state with few or no

unpaired electrons $\mathrm{s}^{34}$. The $2 e \mathrm{CBO}$ amplitude decreased above $250 \mathrm{mK}$, changing to $1 e$ periodicity, due to thermally excited quasiparticles ${ }^{34}$. The low noise $\mathrm{CBO}\left(5 \times 10^{-4} \mathrm{e} / \sqrt{\mathrm{Hz}}\right.$ at $100 \mathrm{~Hz}$ ) were stable for long periods. The symmetric gate electrodes G1 were strongly coupled with a $1 e \mathrm{CBO}$ period of $6.6 \mathrm{mV}$. The asymmetric gate electrode $\mathrm{G} 2$ was weakly coupled with a period of $32.8 \mathrm{mV}$.

\section{CHARGE OFFSETS}

The source-drain current $I_{\mathrm{SD}}$ exhibited sudden jumps when sweeping a gate voltage, equivalent to $\mathrm{CBO}$ phase discontinuities. Fig. 3 shows one such jump which occurred at a voltage $V_{+}$when sweeping the gate more positive and at $V_{-}$when sweeping the gate negative. These were observed for both $1 e$ and $2 e$ oscillations and were induced by sweeping each of the gate electrodes. These jumps indicate sudden charge offsets in the induced charge in the SET island.

The use of both $1 e$ and $2 e \mathrm{CBO}$ revealed an unexpected result. The magnitudes of some of the charge offsets were much larger than expected and are very interesting. If we used only the $1 e \mathrm{CBO}$, as in most previous experiments ${ }^{6,7}$, we would interpret the jump size in Fig. 3(a) as $-0.08 e$. But the $2 e \mathrm{CBO}$ show that the charge shift is actually $+0.92 \pm$ $0.02 e$. An exact $1 e$ charge shift would correspond to a parity shift in the superconducting island due to the addition of an extra quasiparticle. But the charge shifts in Fig. 3(a) are not precisely $1 e$. The sign of the induced charge is equivalent to an excess electron brought close to the island, inducing a reduced positive charge $\Delta Q=c_{1} e /\left(c_{1}+c_{2}\right)$ where $c_{1}$ and $c_{2}$ are the capacitive couplings of the electron to the SET island and elsewhere. 
Over a large gate sweep of $1500 \mathrm{mV}$, up to 14 similar jumps occurred, as shown in Fig. 4, with a distribution of charge offsets $\Delta Q$ from $0.80 e$ to $1.00 e$ and a mean of 0.90 e. Random 1e jumps were observed previously in an NSN device ${ }^{35}$ (using $2 e \mathrm{CBO}$ ) and were interpreted as the tunneling of electrons from the superconducting SET island to nearby traps. We suggest that there are one or more quasiparticle traps close to the SET island. As the gate voltage is swept, single electrons tunnel into the trap or traps giving a charge offset. An obvious possibility would be a small metallic Al particle close to the SET island whose occupancy is limited by Coulomb blockade, as suggested by Brown, Sun and Kane ${ }^{7}$, though the voltage and charge sequences of jumps in Fig.4 are not regular.

Beyond each transition, the $\mathrm{CBO}$ were again stable until the gate voltage was reversed, and the charge offset was reset, though with some hysteresis in gate voltage. Each reversible pair of transitions corresponds to single electron capture and escape from a trap close to the SET island. By convention, we assume that sweeping a gate positive fills the trap with an electron ("capture") while sweeping the gate negative empties the trap ("escape").

As described in the Introduction, such hysteretic charge offsets are common features of metallic SETs, along with two-level fluctuators (TLF). The difference here is that the magnitude of the charge offsets were close to $1 e$, though this would not have been detected using 1e periodic CBOs alone.

Some of these transition pairs were reproducible and enabled us to investigate the detailed dynamics of the charge transfers. One gate voltage was swept slowly, $\mathrm{d} V_{\mathrm{G}} / \mathrm{d} t=$ $\pm a$ (typically $a=36 \mathrm{mV} / \mathrm{s}$ ), keeping all other potentials constant, up and down through the hysteresis transition region and the voltages $V_{i^{+}}$and $V_{i-}$ at which the jumps occurred were recorded. This was repeated for $i=1$ to $N$ times (typically $N=1000$ ) for a given temperature and for a given set of gate voltages, to give good statistics. Fig. 5 shows 10 repeats of one specific hysteresis pair. In each case the charge offset was the same but the capture and escape transition voltages $V_{i^{+}}$and $V_{i-}$ show a distribution of values. Fig. 6 shows scatter plots of many individual sweeps for a capture/escape pair at 25, 100 and $250 \mathrm{mK}$. Each point shows the voltages $V_{i+}$ and $V_{i-}$ and the hysteresis $\Delta V_{i \pm}=V_{i+}-V_{i-}$ at which the jumps occurred during a single sweep. The sweeps take several hours to 
complete in total at each temperature, while data at different temperatures was sometimes taken on different days.

The data is remarkably stable, in that the average jump voltage remains constant for many hours, though sometimes a slight linear drift is observed, or small steps occur in the average value. The drift in the average trap energy can be much less than $k T$ when the conditions are kept constant. But thermal cycling, for instance, can produce changes in the average values of $\left\langle V_{i^{+}}\right\rangle$and $\left\langle V_{i-}\right\rangle$, which can be seen in Fig. 6 by comparing different temperatures. But the scatter plots of the hysteresis remain consistent over long periods of time and the average hysteresis $\Delta V_{ \pm}=\left\langle V_{i^{+}}-V_{i-}\right\rangle=59 \mathrm{mV}$ is much less susceptible to thermal cycling.

Note that the scatter plots are asymmetric, particularly at the lowest temperature. There is a threshold voltage, followed by a long tail. The scatter plots are also temperature dependent, becoming broader as the temperature increases, which is a key part of the analysis below. The standard deviation $\sigma(T)$ increases almost linearly with temperature. For each data set, the variance $\sigma^{2}(T)$ of the hysteresis is equal to the sum of the variances of the capture and escape jumps, within a few percent. This shows that the capture and escape jumps are statistically independent.

\section{EXPONENTIAL DECAYS}

Using the scatter plots we can directly obtain the probability $P$ of the initial state at a voltage $V$ during a sweep by plotting $P=1-i / N$ versus $V_{i}$, as plotted in Figs. 7 and 8 for both electron capture and escape for a range of temperatures. The initial state is stable until some threshold voltage, beyond which it becomes metastable and charge transfer occurs. The transitions become sharper as the temperature falls.

It is immediately clear that the long tails correspond to an exponential decay as the voltage is swept past a threshold voltage $V_{0}$ at $t_{0}$ with $V(t)-V_{0}= \pm a\left(t-t_{0}\right)$ :

$$
P(V)=P\left(V_{0}\right) \exp \left(-\left(t-t_{0}\right) / \tau\right)=\exp \left(-\left|V-V_{0}\right| / a \tau_{0}\right)
$$

where $V_{0}=V_{+}$or $V_{-}$, depending on the sweep direction. Values for the relaxation time $\tau_{0}$ can be obtained using Eq.(3) from the data in Figs. 7 and 8 and are shown in Fig. 9. These times are very long, in electronic terms, and are only slightly temperature dependent. Note that the scatter in the values is much greater than the individual uncertainties in the data analysis. We suggest that the time constant is a tunnelling time 
associated with the trap. This decay will be exponentially sensitive to the local environment and could change slightly on thermal cycling, as did the threshold voltage. The capture and escape times at a given temperature are comparable for data taken on the same day. Exponential decay during a voltage sweep is seen in almost all of our data, though in few cases the smooth decay is masked by drift during the quite lengthy experiments, or by a small admixture of a shifted threshold.

A clear experimental result is that in the low temperature limit, there is a threshold voltage $V_{+}$for electron capture (sweeping one gate voltage $V_{\mathrm{G}}$ positive, with the other electrodes kept constant) and a different threshold $V_{-}$for electron escape (sweeping $V_{\mathrm{G}}$ negative). The control parameters for each transition are $v_{+}=V_{\mathrm{G}}-V_{+}$and $v_{-}=V_{-}-V_{\mathrm{G}}$, as $V_{\mathrm{G}}$ is swept. Hence $v<0$ corresponds to voltages before the threshold is reached and $v$ $>0$ to voltages beyond the threshold. The hysteresis for each pair of transitions is $\Delta V_{ \pm}=$ $V_{+}-V_{-}$. For $v>0$, the metastable initial state decays exponentially as the voltage is swept past the transition threshold. Although this seems like a straightforward result, further discussion is given below.

In a second series of experiments, we measured the real-time capture and escape rates, one electron at a time, by rapidly changing the gate voltage $V_{\mathrm{G} 2}$ from below a threshold $(v<<0$, stable initial state) to a constant value above the threshold $(v>0$, the now metastable initial state decays) and then directly measuring the time before the trap filled or emptied, as seen by a jump in $I_{\mathrm{SD}}$. The trap was reset by sweeping $V_{\mathrm{G} 2}$ back to the stable state below the threshold. Fig. 10 shows a set of 9 repeated measurements at 25 $\mathrm{mK}$. At $t=0$, the gate voltage was rapidly changed through a charge transition region. The measured current $I_{\mathrm{SD}}$ comes to a new equilibrium within $3 \mathrm{~ms}$, but the initial state is now metastable and decays by charge transfer. For the data shown this occurred at a distribution of times from $5 \mathrm{~ms}$ to $28.6 \mathrm{~ms}$. The measurement was repeated to generate an ensemble of switching times $t_{i}(i=1$ to 1000). As with the voltage sweeps, we can obtain the probability $P$ of the initial state at a time $t$ by plotting $P=1-i / N$ versus $t_{i}$, as shown in Fig. 11.

Both the capture and escape rates show excellent exponential decays $\exp (-t / \tau)$ with a decay time $\tau$. Fig. 12 shows the transition rates $1 / \tau$ versus the gate voltage $V_{\mathrm{G} 2}$ at $25 \mathrm{mK}$, in both the electron capture and escape regions. Hysteresis occurs between $V_{-}$and 
$V_{+}$, with no charge movement $(1 / \tau=0)$. The data is asymmetric. A region of constant $1 / \tau$ occurs on both sides of the hysteresis region (dashed lines), but then $1 / \tau$ increases rapidly, possibly to a series of plateaus.

The basic conclusion to this section is that by changing the gate voltage through a threshold voltage, stable charge states become metastable, which then decay exponentially by charge transfer. This was done both by slowly sweeping a gate voltage through a threshold and also by rapidly crossing a threshold to a fixed voltage and measuring the decay in real time. Although the decay time $\tau$ is reproducible for a given experiment, it can change significantly, after thermal cycling for instance. The capture and escape times are almost independent of temperature, Fig. 4 , and are long, $\approx 30 \mathrm{~ms}$, suggesting that the threshold is the onset of energetically allowed tunneling. Similar results are obtained from both parts of the hysteretic transition. By comparison, Buehler et $a l .{ }^{36}$ measured the telegraph noise for a two-level fluctuator in an $r f$-SET with a switching time of $3.4 \mu$ s. In general, a wide range of possible decay times are observed in TLFs.

\section{EXCITATION OF METASTABLE STATES}

As described above, each transition has a sharp threshold at low temperatures which broadens as $T$ increases. The rate of decrease of the initial state probability $P(V)$ at a voltage $V$ is $(1 / P) \mathrm{d} P / \mathrm{d} t=a \mathrm{~d} \ln P / \mathrm{d} v=-1 / \tau$. The decay time $\tau(V)$ at a given voltage can be obtained by numerical differentiation of each $\log _{10} P$ vs. $V$ plot, as shown in Fig. 13 at $25 \mathrm{mK}$, for both electron capture and escape. In both cases, the decay time decreases exponentially as the threshold is approached, and then becomes constant beyond the threshold as discussed above. Below each threshold, an Arrhenius factor ${ }^{9}$ gives an excellent account of the escape and capture rates with an excitation energy $E=e|v| / \gamma$ which goes to zero at the threshold $(v=0)$. The factor $\gamma$ is a geometrical voltage scaling factor $^{37}$, which relates the voltage applied to the gate electrode to the excitation energy required for the trap, as in Eqs. (1) and (2). Experimentally, an excellent fit is found for

$$
\begin{array}{ll}
1 / \tau(v, T)=\left(1 / \tau_{0}\right) \exp (-E / k T) & \text { for } v<0 \\
1 / \tau(v, T)=1 / \tau_{0} & \text { for } v \geq 0
\end{array}
$$

where $1 / \tau_{0}$ is the value at and just above the threshold, for both electron capture and escape. This result is confirmed at other temperatures, as shown at $242 \mathrm{mK}$ in Fig. 14. 
The solid lines show the exponential fit to the data before the threshold. In both these examples, the constant decay times beyond the thresholds (dashed lines) are approximately the same for both electron capture and escape.

The hysteresis separates the escape and capture processes as the gate voltage is swept. Without the hysteresis, this would presumably act as a two-level fluctuator (TLF) switching back and forth. If the TLF is in thermal equilibrium, then the populations of the 2 states would be related by a Boltzmann factor, $N_{1} / N_{2}=\exp (-E / k T)$. Detailed balance between the capture and escape rates then gives $N_{1} / \tau_{1}=N_{2} / \tau_{2}$, so that

$$
\tau_{1}(v, T) / \tau_{2}(v, T)=\exp (e v / \gamma k T)
$$

as in Eq.(2). By shifting the voltages so that the escape and capture voltage thresholds coincide, we can plot $\tau_{1} / \tau_{2}$ versus $\Delta V$ as shown in Fig. 15 for $25 \mathrm{mK}$. The ratio of the decay times is exponential, Eq.(5), over 5 decades. This clearly shows that the basic charge transfer is driven by thermal excitation between two states in thermal equilibrium, despite the hysteresis and the long decay times involved.

Eq.(4) can be integrated to give the probability $P(v, T)$ of the initial state during a voltage sweep:

$$
\begin{array}{ll}
P(v, T)=\exp \left[\left(-\gamma k T / e a \tau_{0}\right) \exp (e v / \gamma k T)\right] & \text { for } v<0 \\
P(v, T)=\exp \left(-\gamma k T / e a \tau_{0}\right) \exp \left(-v / a \tau_{0}\right) & \text { for } v \geq 0
\end{array}
$$

where $v=V_{\mathrm{G}}-V_{+}$or $V_{-}-V_{\mathrm{G}}$ as appropriate. Fits to Eq.(6) are plotted in Fig. 16 for capture and escape transitions at 25 and $165 \mathrm{mK}$. Figs. 16(a) and 16(d) show the general fit while Figs. 16(b) and 16(e) plot $\log (P)$ to show the exponential decays after the threshold. The nested exponentials in Eq.6(a) give a strong temperature dependence for $v$ $<0$. A plot of $\log _{10}\left(-\log _{10} P\right)$ versus $v$ gives a straight line, before the threshold, with a slope proportional to $1 / \gamma T$, as shown in Figs. 16(c) and 16(f). The fits are excellent, using $V_{+}$or $V_{-}$as fitting parameters, with only small deviations for small values of $P$ and $1-P$.

The product $\gamma T$ was also used as a fitting parameter in Eq.(6) since $\gamma$ may be temperature dependent and the effective electron temperature $T$ may be larger than the dilution refrigerator temperature, due to Joule heating. The experimental temperature dependence of $\gamma T$ was reported previously by us $^{10}$ for the electron escape data. Above 50 $\mathrm{mK}, \gamma T$ was proportional to $T$ with $\gamma=145 \pm 5$. Below $50 \mathrm{mK}, \gamma T$ lay above this line. We 
assumed that $\gamma$ was constant, but that the electronic temperature of the SET was increased due to Joule heating. The thermal conductance from an SET island ${ }^{38}$ typically varies as $\left(T_{\mathrm{SET}}{ }^{5}-T^{5}\right)$. Hence the SET electron temperature reached a minimum value of $\sim 35 \mathrm{mK}$. Note that the temperatures given for the various data sets refer to the refrigerator temperature and that $25 \mathrm{mK}$ fridge temperature corresponds to an electron temperature of about $36 \mathrm{mK}$, while a fridge temperature of $50 \mathrm{mK}$ corresponds to about $52 \mathrm{mK}$. Above $50 \mathrm{mK}$, the Joule heating should be minimal (but see below).

Fig. 18 shows all our data for $\gamma T$, which confirms our previous result but the electron capture data has rather more scatter than the escape data. One reason for this is that in some cases a small fraction, $\leq 5 \%=(1-\alpha)$, of the transitions in the voltage sweeps exhibit a threshold which is shifted by about $5 \mathrm{mV}=\Delta v$. The probability of the initial state is then given by

$$
P_{\alpha}(v, T)=\alpha P(v, T)+(1-\alpha) P(v+\Delta v, T)
$$

This probably comes from another weakly coupled TLF in the system which shifts the background potential slightly, and which occurs near the gate voltage range for electron capture. Two of these data sets also show relatively high values of $\gamma T$, which were again associated with a TLF, which is equivalent to introducing noise in the gate voltage and smearing out the transition region.

The conclusion from this section is that before the threshold is reached, Arrhenius thermal excitation occurs. This is not the more usual thermal activation over a potential barrier, but excitation up to a tunneling threshold. The exponential ratio of the capture and escape times show that it is equivalent to the process of reaching thermal equilibrium between two coupled energy levels.

\section{TRAPPING MODEL}

These results give a very clear picture of the dynamics of trapped electrons in a superconducting SET. There are four distinct phenomena to be explained: charge offsets close to $1 e$ in magnitude; hysteresis; constant decay times beyond a threshold voltage and thermally excited decay times below the threshold. All these results are consistent with previous experiments - the new aspects here are the use of a SCPT to show charge offsets close to $1 e$ and the detailed measurements of the decay dynamics. 
Three generic types of defects may be relevant here ${ }^{26}$. These are (a) bistable twolevel systems (TLS) or charge fluctuators (TLF) ${ }^{6}$, (b) single electron traps, including surface states, and (c) small metallic particles, whose charge will be limited by Coulomb blockade $^{7}$. The latter two could be charged by tunneling from an SET electrode or the SET island. All three types might be coupled together by Coulomb interactions or by lattice strains. But it is notoriously difficult to identify specific defects from measurements of the trap dynamics!

The large magnitude of the charge offsets, $\sim 1 e$, immediately seems to rule out a single TLF, as in (a), in which charge oscillates between two localised sites. Even charge offsets of $\leq 0.2 e$, observed previously with $1 e$ periodic CBOs, are difficult to understand. Tunneling from the SET island or an electrode to a trapped site, as in (b), could give a charge offset close to $1 e$. Brown, Sun and $\mathrm{Kane}^{7}$ suggested that small isolated Al grains could act as quasiparticle traps, as in (c). These grains do seem to be present in many metallic SETs, including some used here, as shown in some SEM images. If one of these grains were coupled by tunneling to the SET island or leads, then it could charge up as the gate voltage is swept, limited by Coulomb blockade. This could explain the "ladder" of charge offsets, close to 1e, shown in Fig. 4. Each extra electron will shift the induced charge on the SET island by less than $1 e$, due to fractional coupling to other electrodes. These large charge offsets would not be seen using $1 e$ periodic CBOs as in most previous experiments. For an ideal system, the Coulomb blockade ladder would be uniform and evenly spaced. But the presence of other coupled defects could produce variations and a range of possible effects as discussed below.

The experimental voltage dependent tunneling rates $1 / \tau$ are revealing. The temperature independence and the long values of the escape and capture times suggest that they are due to tunneling between defect states. The ratio of the escape and capture times follow a Boltzmann factor, Fig. 15, showing that the charge states are in thermal equilibrium.

Electron tunneling from a superconductor to small metallic particles was studied by Zeller and Giaever ${ }^{39}$ and through individual Al particles by Ralph, Black and Tinkham ${ }^{40}$. The tunneling rate $1 / \tau$ versus voltage is proportional to the superconducting density of states, and diverges at the energy gap. If the transitions in Fig. 3 are tunneling 
to or from the superconducting island, then we should observe a maximum of $1 / \tau$ for $v \geq$ 0 , rather than the observed constant $1 / \tau_{0}$ seen in Figs. 13 and 14. This should apply to tunnelling from the superconductor island to a superconducting grain, to a normal metal particle or to an isolated single-electron trap. This is clearly not observed experimentally here.

The hysteresis $\Delta V_{ \pm} / \gamma=59 \mathrm{mV} / 145=407 \mu \mathrm{V}$ is quite close to the superconducting energy gap $2 \Delta / e=360 \mu \mathrm{V}$. Conversely, hysteresis in TLFs in previous experiments ${ }^{6}$ had a wide range of values and was the same in both the normal and superconducting states, though the origin of the hysteresis was not explained. It is most probable that the hysteretic behaviour in these new experiments has the same origin.

Neither a single TLF nor a single metallic grain seem able to explain our results.

But there is considerable experimental evidence that trap-trap interactions are very important in SETs and other tunnelling devices which exhibit TLF behaviour, leading to the correlated switching of clusters of traps. Also the relaxation rates we observe are consistent with those for a TLF and Eq.(4) was explicitly used by Grupp et al. to describe the relaxation of a single TLF coupled to a metallic charge trap. We therefore suggest the following to explain (i) charge offsets close to 1e, (ii) hysteresis and (iii) Arrhenius excitation. As any gate voltage $V_{\mathrm{G}}$ is swept, intrinsic TLF transitions will occur, with local charge movements ${ }^{6}$, but relatively small charge offsets, $|\Delta Q|<0.1$ e. But each transition will change the Coulomb energy of other nearby traps and may trigger the tunneling of an electron between the SET island and a quasiparticle trap, which may then stabilise the TLF system, giving hysteresis.

A similar mechanism was proposed by Rogers et al. ${ }^{41,42}$ for electron traps in amorphous Si. They used a double-well potential to model traps, based on the ionicreconfiguration model used to describe the low-temperature properties of glasses. They stressed that the rate-limiting process is a reversible displacement or reconfiguration of ions forming a trap. At low temperature this would proceed by ionic-configurational tunneling in which, for instance, an oxygen vacancy tunnels between sites. In itself, this would not produce any significant charge offset. But, the ionic rearrangement could then trigger a change in the trapped charge, which in turn affects the configuration as the ions relax, lowering the energy. In their model an occupied trap has an energy less than the 
chemical potential $\mu$, but when it is empty, the energy is above the chemical potential. Hence, as the ions make thermally excited or tunneling transitions between the configurations, the trap will charge or discharge by electron tunneling from a nearby conductor, in a two-stage process. The shift in energy leads directly to hysteresis. In this case the trap is directly created by the ionic TLF. However, other experiments ${ }^{43}$ suggested that ionic reconfiguration played only a secondary role in a small number of defects and that electron tunneling played the major role in most TLFs. Hysteresis could also arise from an atomic rearrangement as an electron tunnels into a trap and the atomic positions relax and dissipate the energy in phonons. However the wide range of hysteresis values found by Furlan and Lotkhov ${ }^{6}$ would suggest that it is not associated with a specific trap configuration.

If the TLFs consist of electrons tunneling between traps, rather than ionic tunneling, then interactions between neighbouring traps can produce similar effects ${ }^{44}$. A simple model can be used to express the hysteresis for both electronic and ionic TLFs. The basic idea is that the switching of a TLF induces the tunneling of an electron, or quasiparticle, into a trap. The excitation energies ${ }^{39}$ of the TLF, $E$, and the trap, $E_{1}$, will depend on $V_{\mathrm{G}}$, the TLF state $(M=0,1)$ and the trap occupancy $(N=0,1)$ as

$$
\begin{aligned}
& E=e\left(V_{+}-V_{\mathrm{G}}\right) / \gamma-N \Delta E \\
& E_{1}=e\left(V_{1}-V_{\mathrm{G}}\right) / \gamma_{1}-M \Delta E
\end{aligned}
$$

where $\gamma_{1}$ is a voltage scaling factor for the trap as $V_{\mathrm{G}}$ is swept and $\Delta E$ is the difference of the Coulomb interaction energy between an electron in the trap and the charged TLF in the $M=0$ and the $M=1$ states. This is shown schematically in Fig.18. As $V_{\mathrm{G}}$ is swept, the trap will fill, or the TLF switch, whenever $E$ or $E_{1}=0$, or thermal excitation occurs. Since both mechanisms are independently thermally excited, the sequence of events will depend on their relative energies and rates. If we start in the state $(M=0, N=0)$ and sweep $V_{\mathrm{G}}$ positive, the TLF will switch at $V_{+}$to state $(1,0)$. If this then makes $E_{1}<0$, the trap will rapidly fill $\left(<<\tau_{0}\right)$ to state $(1,1)$, giving $|\Delta Q| \approx 1 e$, but also decreasing $E$ and stabilising the TLF. Sweeping $V_{\mathrm{G}}$ back negative, the TLF switches to state $(0,1)$ at $V_{-}$, triggering the trap emptying back to $(0,0)$. The voltage hysteresis $\Delta V_{ \pm}=\gamma \Delta E / e$. Thermal excitation will occur as a threshold is approached. 
The relaxation of a trap or TLF after switching or tunneling can be described by introducing terms of the form $-M \Delta E_{0}$ and $-N \Delta E_{1}$ in Eq.8(a) and Eq.8(b) respectively, where $\Delta E_{0}$ and $\Delta E_{1}$ are the energy losses on relaxation. These terms would give rise to hysteresis in trap occupancy for a single trap or TLF without any defect interactions.

This model may also explain some of the data in Fig. 12 if the TLF switching time was much longer than the trap tunneling time ${ }^{41}$. In Fig. 12, we started in the state $(0$, 0 ) and quickly changed to a new constant voltage $V_{\mathrm{G}}$, in a time shorter than the TLF switching time, and measured $1 / \tau$. For $V_{\mathrm{G}}<V_{2}$, the TLF will switch first. But if $V_{\mathrm{G}}>V_{2}$ the trap can fill first to the state $(0,1)$, followed by the TLF switch to $(1,1)$. On the reverse sweep, the trap can empty first if $V_{\mathrm{G}}<V_{1}=V_{2}-\gamma_{1} \Delta E / e$ to the state $(1,0)$ and then to the state $(0,0)$. If the trap tunneling rate is faster than the TLF switching time, a sudden increase in the transition rates will be seen at $V_{1}$ and $V_{2}$, as in Fig. 12. Note that in a slow gate sweep, no transitions were observed at the voltages $V_{1}$ and $V_{2}$. The voltage scaling values $\gamma=145$ and $\gamma_{1}=\gamma\left(V_{2}-V_{1}\right) /\left(V_{+}-V_{-}\right)=860$ suggest that the TLF and the trap are close to the SET island ${ }^{6,7}$. We are not able to resolve the details of the faster quasiparticle trapping process. However, Aumentado et al. ${ }^{45}$ have shown that, even in a specially designed SET, some quasiparticle poisoning will occur in the even parity ground state of the SET island. Over the long time scales in our experiment, single quasiparticles will become trapped when it is energetically favorable.

In any given SET, the specific behaviour observed will depend on the distribution of actual defects and traps and the coupling between them as well as the relative decay times. In the experiments reported here, only one pair of hysteretic transitions were sufficiently stable to produce reproducible and reliable data with timescales within the observable range.

\section{CONCLUSIONS}

In summary, we have measured the capture and escape of trapped electrons in an SET using both $1 e$ and $2 e \mathrm{CBO}$ to measure the charge offsets. Unexpectedly large charge offsets close to $1 e$, thermal excitation to a tunneling transition and hysteresis between metastable states were observed. Detailed measurements of the capture and escape rates enabled the dynamics of the trapping process to be investigated. Allowing for the hysteresis, the metastable states are in thermal equilibrium, with relative populations 
given by a Boltzmann factor. Neither a single TLF nor a single electronic trap seem able to explain our results. We suggest that both quasiparticle traps and two-level fluctuators contribute to intrinsic charge movement in SCPTs, and that electrostatic coupling between them can produce correlated charge movements and hysteresis. This model is consistent with, and may help to explain, previous experiments ${ }^{6,7}$. An understanding of these defects is crucial if the potential of charge sensitive detectors such as the SET and the SCPT is to be achieved.

\section{ACKNOWLEGEMENTS}

We thank Denis Vion and Mark Dykman for discussions and F.Greenough, A.K.Betts and others for technical support. PJM gratefully acknowledges the hospitality and stimulation provided by Group Quantronique, CEA Saclay. The work was supported by the EPSRC and by Royal Holloway, University of London.

${ }^{1}$ L. C. L. Hollenberg et al. Phys.Rev. B 69, 113301 (2004).

2 G. Papageorgiou et al., Appl.Phys.Lett. 86, 153106 (2005); E. Rousseau, Y. Mukharsky, D. Ponarine, O. Avenel and E. Varoquaux, J. Low Temp. Phys. 148, 193 (2007); E. Rousseau, D.Ponarin, L.Hristakos, E.Varoquaux, O.Avenel and Y.Mukharsky, arXiv:0808.0955v1.

${ }^{3}$ S. J. Angus, A. J. Ferguson, A. S. Dzurak and R. G. Clark, Appl.Phys.Lett. 90, 112103 (2008).

${ }^{4}$ D. Vion, A. Aassime, A. Cottet, P. Joyez, H. Pothier, C. Urbina, D. Esteve and M. H. Devoret, Science 296, 886 (2002).

${ }^{5}$ D. E. Grupp, T. Zhang, G. J. Dolan and N. S. Wingreen, Phys.Rev.Lett. 87, 186805 (2001).

${ }^{6}$ M. Furlan and S. V. Lotkhov, Phys.Rev. B 67, 205313 (2003).

${ }^{7}$ K. R. Brown, L. Sun and B. E. Kane, Appl.Phys.Lett. 88, 213118 (2006). 
${ }^{8}$ G. Ithier, E. Collin, P. Joyez, P. J. Meeson, D. Vion, D. Esteve, F. Chiarello, A.

Shnirman, Y. Makhlin, J. Schriefl, and G. Schön, Phys.Rev. B 72, 134519 (2005).

${ }^{9}$ C. T. Rogers and R. A. Buhrman, Phys.Rev.Lett. 53, 1272 (1984).

${ }^{10}$ D. G. Rees, P. Glasson, L. R. Simkins. E. Collin, V. Antonov, P. G. Frayne, P. J. Meeson and M. J. Lea, Appl.Phys.Lett. 93, 173508 (2008).

${ }^{11}$ K. S. Ralls, W. J. Skocpol, L. D. Jacel, R. E. Howard, L. A. Fetter, R. W. Epworth and D. M. Tennant, Phys.Rev.Lett. 52, 228 (1984).

${ }^{12}$ D. H. Cobden, M. J. Uren and M. Pepper, Phys.Rev.Lett. 71, 4230 (1993).

${ }^{13}$ H. Grabert and U. Weiss, Phys.Rev.Lett., 54, 1605 (1985).

${ }^{14}$ G. Zimmerli, T. M. Eiles, R. L. Kautz and J. M. Martinis, Appl.Phys.Lett. 61, 237 (1992).

${ }^{15}$ A. B. Zorin, F.-J. Ahlers, J. Niemeyer, T. Wiemann, H. Wolf, V. A. Krupenin, and S. V. Lotkov, Phys.Rev. B53, 13682 (1997).

${ }^{16}$ A. N. Tavkhelidze and J. Mygind, J.Appl.Phys. 83, 310 (1998).

${ }^{17}$ N. M. Zimmerman, J. L. Cobb and A. F. Clark, Phys.Rev. 56, 7675 (1997).

${ }^{18}$ V. A. Krupenin, D. E. Presnov, M. N. Savvateev, H. Scherer, A. B. Zorin and J.

Niemeyer, J.Appl.Phys. 84, 3212 (1998).

${ }^{19}$ M. Kenyon, J. L. Cobb, A. Amar, D. Song, N. M. Zimmerman, C. J. Lobb and F. C. Wellstood, IEEE Transactions on Applied Superconductivity 9, 4261 (1999).

${ }^{20}$ T. Fujisawa and Y. Hirayama, Appl.Phys.Lett. 77, 543 (2000).

${ }^{21}$ W. H. Huber, S. B. Martin and N. M. Zimmerman, in Experimental Implementation of Quantum Computation (IQC'01), edited by R. Clark (Rinton, Princeton, NJ, 2001). 
${ }^{22}$ N. J. Zimmerman, W. H. Huber, A. Fujiwara and Y. Takahashi, Appl.Phys.Lett. 79, 3188 (2001).

${ }^{23}$ N. J. Zimmerman, B. J. Simonds, A. Fujiwara, Y. Ono, Y. Takahashi and H. Inokawa, Appl.Phys.Lett. 90, 033507 (2007).

${ }^{24}$ D. E. Grupp, T. Zhang, G. J. Dolan and N. S. Wingreen, Phys.Rev.Lett. 87, 186805 (2001).

${ }^{25}$ T. M. Buehler, D. J. Reilly, R. P. Starrett, V. C. Chan, A. R. Hamilton, A. S. Dzurak and R. G. Clark, J.Appl.Phys. 96, 6827 (2004).

${ }^{26}$ N. M. Zimmerman, W. H. Huber, B. Symonds, E. Hourdakis, A. Fujiwara, Y. Ono, Y. Takahashi, H. Inokawa, M. Furlan and M. W. Keller, J.Appl.Phys. 104. 033710 (2008).

${ }^{27}$ Quantum Tunneling in Condensed Media, (Eds. Yu. Kagan, A. J. Leggett, Elsevier, 1992)

${ }^{28}$ M. I. Dykman, I. B. Schwartz and M. Shapiro, Phys.Rev. E72, 021102 (2005).

${ }^{29}$ S. Shulz, S. Schnüll, C. Heyn and W. Hansen, Phys.Rev. B 69, 195317 (2004).

${ }^{30}$ D. G. Rees, PhD thesis, University of London (2006).

${ }^{31}$ The SET parameters are: normal state resistance $R_{\mathrm{N}}=30 \mathrm{k} \Omega$; Coulomb energy $E_{\mathrm{C}}=35$ $\mu \mathrm{eV}$; Josephson energy $E_{\mathrm{J}}=20 \mu \mathrm{eV}$; energy gap $2 \Delta=380 \mu \mathrm{eV}$.

${ }^{32}$ L. R. Simkins. PhD thesis, Royal Holloway, University of London, 2009.

${ }^{33}$ P.-M. Billangeon, F. Pierre, H. Bouchiat, R. Deblock, cond-mat/0607639v3 (2006).

${ }^{34}$ A. Amar, D. Song, C. J. Lobb and F. C. Wellstood, Phys.Rev.Lett. 72, 3234 (1994).

35 T. M. Eiles, J. M. Martinis and M. H. Devoret, Phys.Rev.Lett. 70, 1862 (1993); T. M. Eiles and J. M. Martinis, Phys.Rev. B 50, 627 (1994). 
${ }^{36}$ T. M. Buehler, V. Chan, A. J. Ferguson, A. S. Dzurak, F. E. Hudson, D. J. Reilly, A. R. Hamilton, R. G. Clark, D. N. Jamieson, C. Yang, C. I. Pakes, and S. Prawer, App.Phys.Lett. 88, 192101 (2006).

${ }^{37}$ In general, $E \propto v^{\xi}$. We find $\xi=1.0 \pm 0.05$. If the tunneling barrier went to zero, we would expect $\xi=1.5$.

${ }^{38}$ A. N. Korotkov, M. R. Samuelsen and S. A. Vasenko, J.Appl.Phys. 76, 3623 (1994).

${ }^{39}$ H. R. Zeller and I. Giaever, Phys.Rev. 181, 789 (1969).

${ }^{40}$ D. C. Ralph, C. T. Black and M. Tinkham, Phys.Rev.Lett. 74, 3241 (1995); ibid, 76, 688 (1996); ibid, 78, 4087 (1997).

${ }^{41}$ C. T. Rogers and R. A. Buhrman, Phys.Rev.Lett. 55,859 (1985).

${ }^{42}$ C. T. Rogers, R. A. Buhrman, H. Kroger and L. N. Smith, Appl.Phys.Lett. 49, 1107 (1986).

${ }^{43}$ X. Jiang and J. C. Garland, Phys.Rev.Lett. 66, 496 (1991).

${ }^{44}$ R. T. Wakai and D. J. Harlingen, Phys.Rev.Lett. 58, 1687 (1987).

${ }^{45}$ J. Aumentado, M. W. Keller, J. M. Martinis and M. H. Devoret, Phys.Rev.Lett. 92, 066802 (2004). 
FIG. 1. SEM micrograph showing (i) the SET source (S) and drain (D) electrodes and island, (ii) the gate electrodes (G1 and G2). The contrast has been increased in the central region to show the Al electrodes. The source, drain and gate leads are covered by a metallic Au ground plane on the upper and lower parts of the image.

FIG. 2. The $I-V$ characteristic of the SET, showing the Cooper pair peak (C), the JQP peak (J) and the quasiparticle branch.

FIG. 3. Coulomb blockade oscillations CBO at $15 \mathrm{mK}$ for up (black, a) and down (red/grey, •) gate sweeps (a) at the JQP peak $\left(V_{\mathrm{SD}}=0.55 \mathrm{mV}\right.$, lower trace) showing $1 e$ periodicity and (b) at the Cooper pair peak $\left(V_{\mathrm{SD}}=25 \mu \mathrm{V}\right.$, upper trace) showing $2 e$ periodicity. The data show a hysteretic transition into a trapped charge state with $\delta Q=0.92 e$. The $y$-axes have been offset for clarity.

FIG. 4. The offset charge $\Delta Q / e$ versus gate voltage $V_{\mathrm{G} 1}$ observed using $2 \mathrm{e}$ periodic CBOs, showing the large charge offsets, $\sim 1 e$.

FIG. 5. Hysteresis in the CBO current while sweeping gate voltage $V_{G 2}$. The transition voltages for electron capture and escape $V_{i+}$ and $V_{i-}$ show a range of values. The vertical axis for each trace is offset for clarity.

FIG. 6. Scatter plots of 1000 individual sweeps for an electron capture/escape pair at 25, 100 and $250 \mathrm{mK}$ refrigerator temperatures. Each point shows the voltages $V_{i+}$ and $V_{i-}$ and the hysteresis $\Delta V_{i \pm}=V_{i+}-V_{i-}$ at which the jumps occurred for a single sweep.

FIG. 7. Probability plots $P(V)$ of the initial state when sweeping the gate positive (electron capture) at 242, 200, 165, 116, 100, 50, 45 and $25 \mathrm{mK}$ refrigerator temperatures. The plots are offset along the $x$-axis for clarity. The temperature decreases from left to right. The lower graph is a logarithmic plot.

FIG. 8. Probability plots $P(V)$ of the initial state when sweeping the gate negative (electron capture) at 242, 200, 165, 116, 100, 50, 45 and $25 \mathrm{mK}$ refrigerator temperatures. The plots are offset along the $x$-axis for clarity. The temperature decreases from right to left. The lower graph is a logarithmic plot. 
FIG. 9. The relaxation times $\tau_{0}$ for electron capture ( $\square$ ) and escape (•) below 250 mK.

FIG. 10. The source-drain current $I_{\text {SD }}$ versus time. At $t=0$, the gate voltage is switched from a stable to a metastable voltage. The change in $I_{\mathrm{SD}}$ due to charge transfer is delayed. Nine data sets are shown at $25 \mathrm{mK}$ refrigerator temperature.

FIG. 11. Typical exponential decays after switching a gate voltage from stable to a metastable voltage, repeated 1000 times, at $25 \mathrm{mK}$ refrigerator temperature. Two data sets are shown, for electron escape $\left(V_{\mathrm{G} 2}=-88 \mathrm{mV}\right)$ and electron capture $\left(V_{\mathrm{G} 2}=\right.$ $+88 \mathrm{mV})$.

FIG. 12. The measured relaxation times when switching a gate voltage rapidly from a stable voltage (in region $A$ ) to a metastable voltage, at $25 \mathrm{mK}$ refrigerator temperature. The voltages $V_{+}$and $V_{-}$show the threshold voltages when sweeping the gate voltage slowly. The voltages $V_{1}$ and $V_{2}$ are discussed in the text.

FIG. 13. The relaxation time $\tau(V)$ for (a) electron escape and (b) electron capture at $25 \mathrm{mK}$ refrigerator temperature.

FIG. 14. The relaxation time $\tau(V)$ for (a) electron escape and (b) electron capture at $242 \mathrm{mK}$.

FIG. 15. The ratio $\tau_{2} / \tau_{1}$ versus the voltage $\Delta V=V-V_{+}$for $\tau_{1}$ and $\Delta V=V-V_{-}$for $\tau_{2}$. The solid line shows the fit to Eq.(2).

FIG. 16. (a, d) $P(V, T)$ for electron capture and escape transitions at 25 and $165 \mathrm{mK}$ refrigerator temperatures, showing fits to Eq.(4). (b, e) $P(V, T)$ for electron capture and escape transitions at 25 and $165 \mathrm{mK}$ on a logarithmic scale, showing fits to Eq.(4) and the exponential decay beyond the transition. (c, f) $-\log [P(V, T)]$ for electron capture and escape transitions at 25 and $165 \mathrm{mK}$, showing the thermal excitation before the threshold.

FIG. 17. The parameter $\gamma T$ versus the refrigerator temperature $T$ for electron escape ( $\square$ and $\bullet$ ) and electron capture ( $\square$ and $\odot)$. The lines show $\gamma T$ (solid) and $\gamma T_{\text {SET }}$ (dotted, allowing for heating ${ }^{38}$ ) for $\gamma=145$. 
FIG. 18. Schematic model, showing a TLF coupled to an electron trap. The voltages $V_{+}$and $V_{-}$show the threshold voltages when sweeping the gate voltage slowly. The voltages $V_{1}$ and $V_{2}$ are discussed in the text. 
Simkins Figure 1

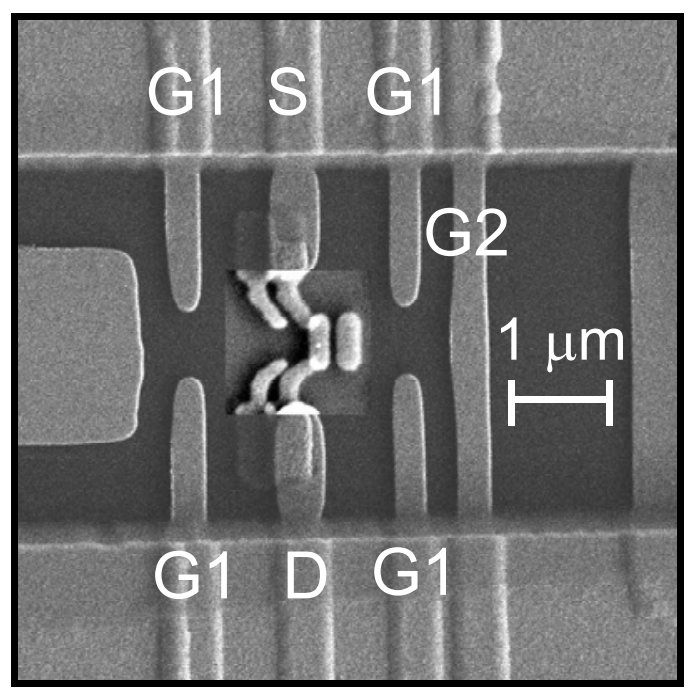


Simkins Figure 2

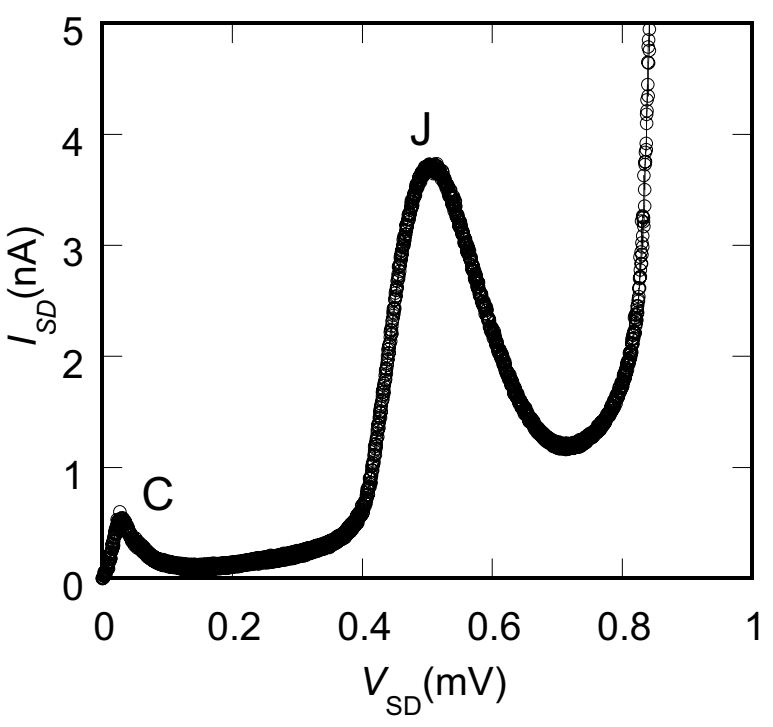




\section{Simkins Figure 3}

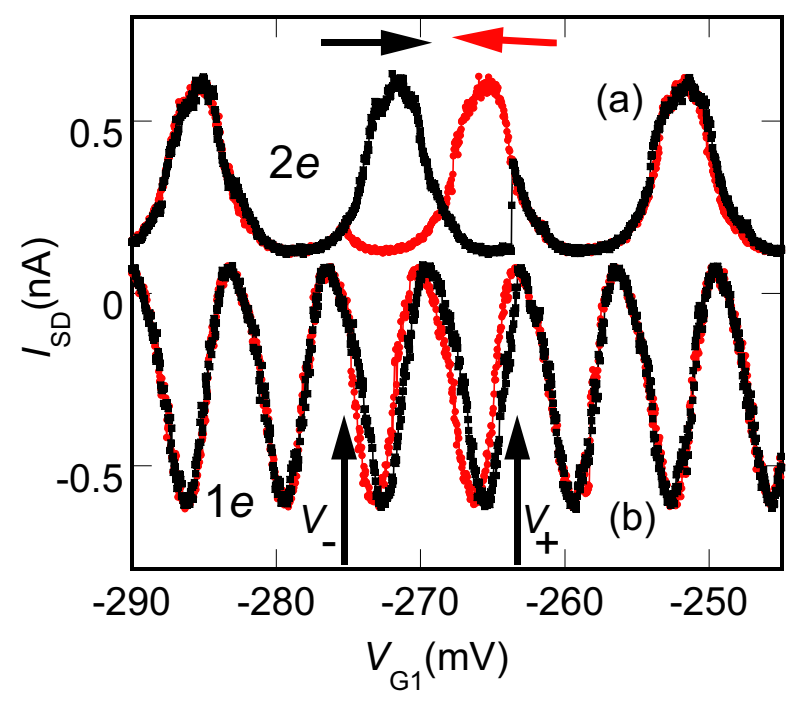


Simkins Figure 4

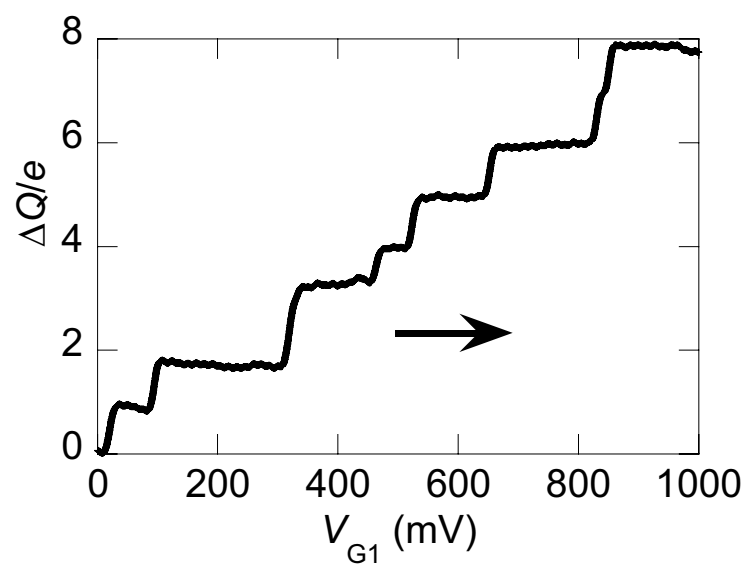


Simkins Figure 5

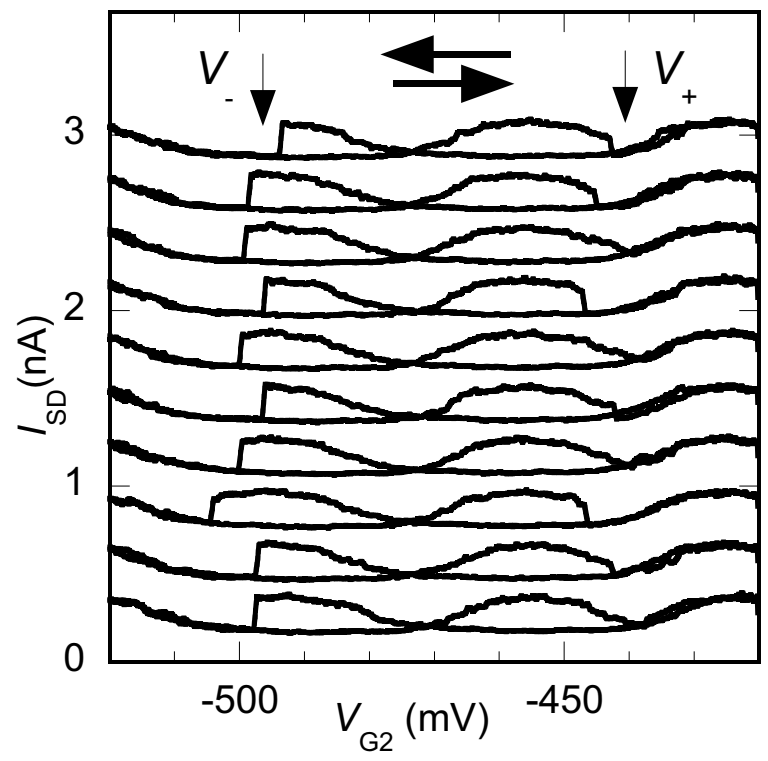




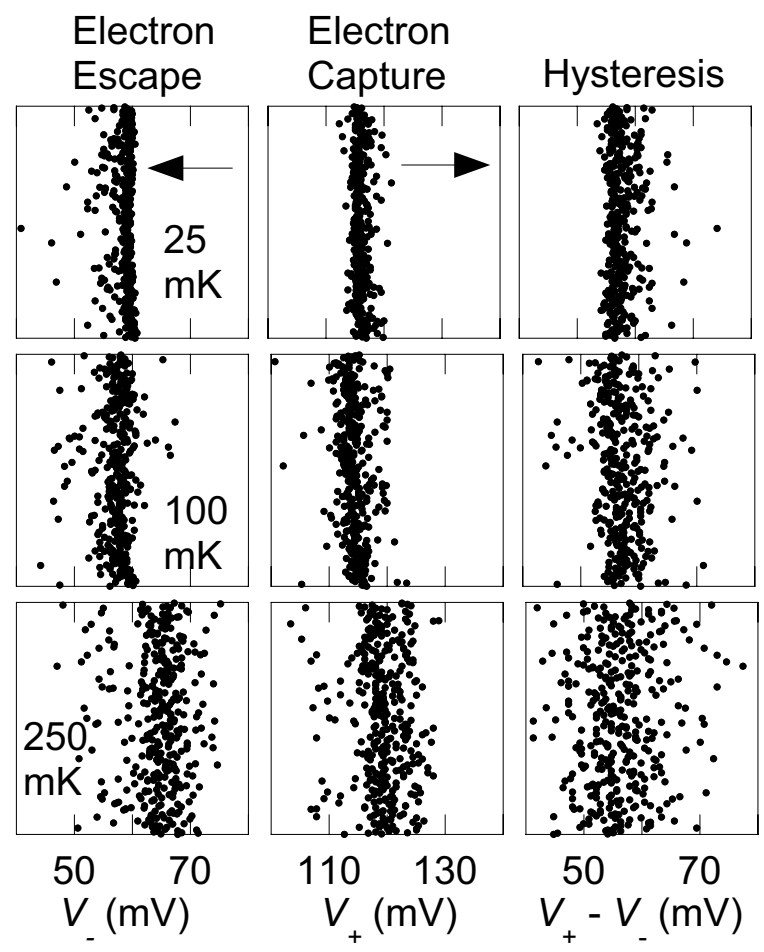

Simkins Figure 6 


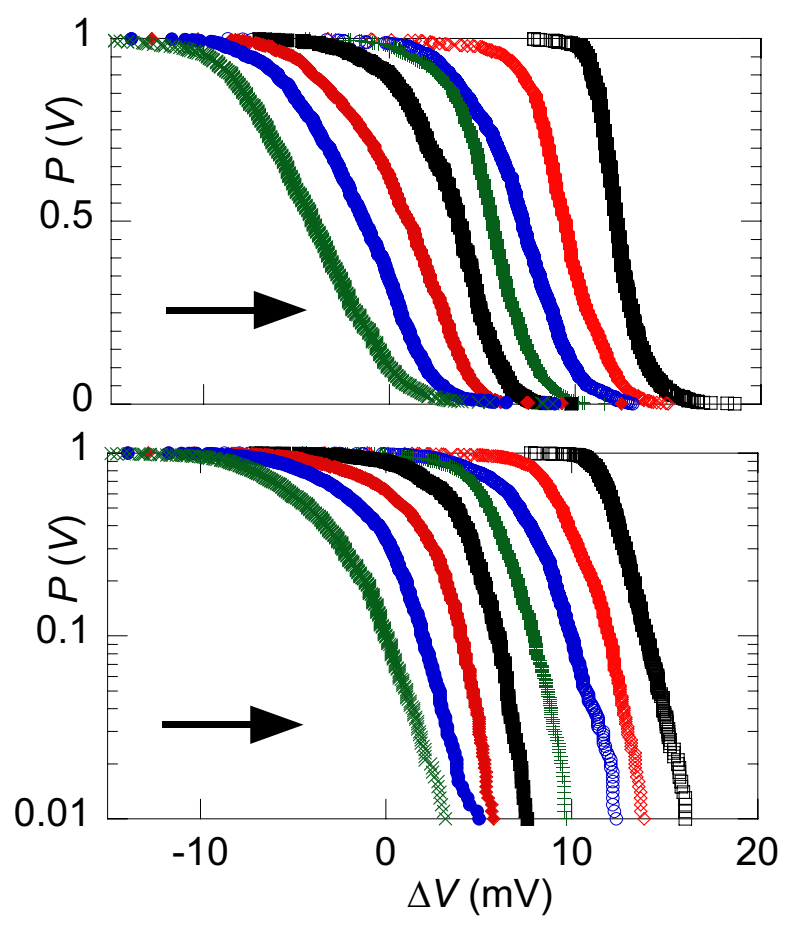

Simkins Figure 7 


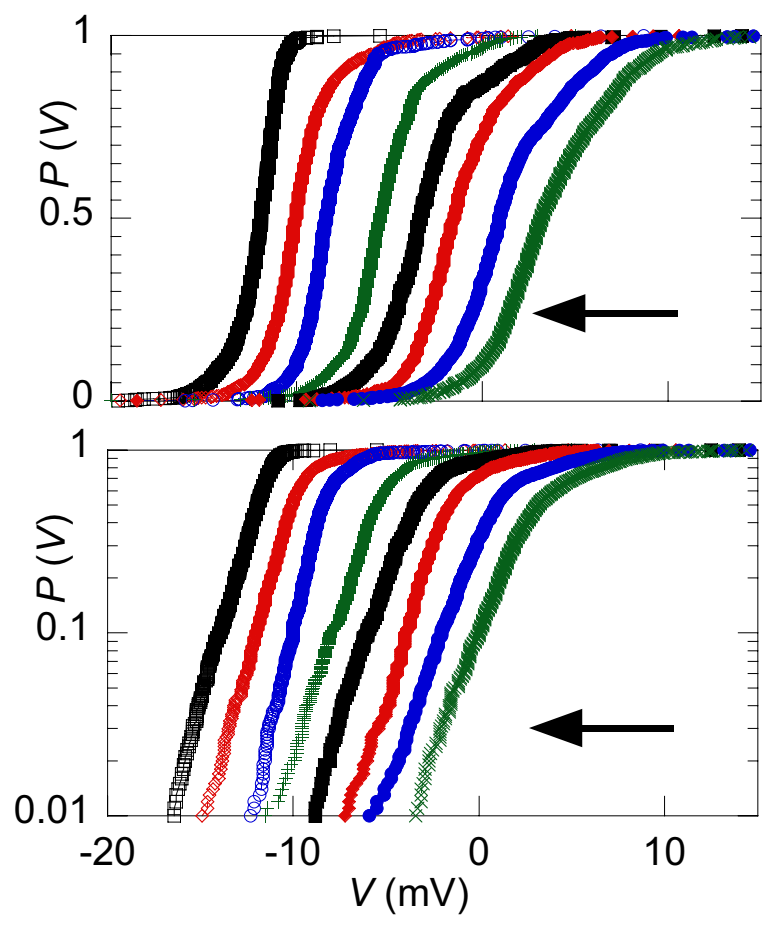

Simkins Figure 8 
Simkins Figure 9

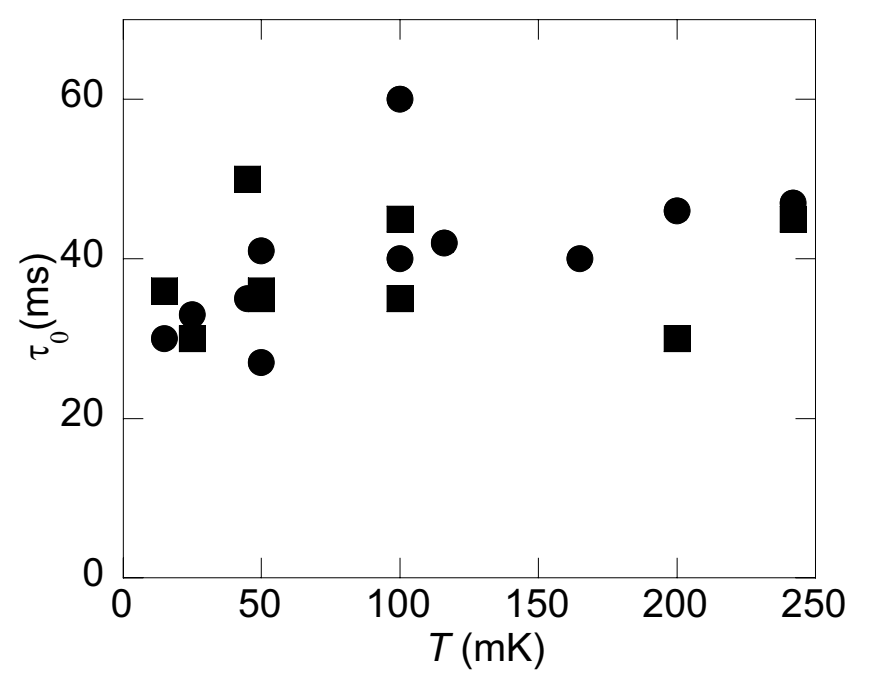


Simkins Figure 10

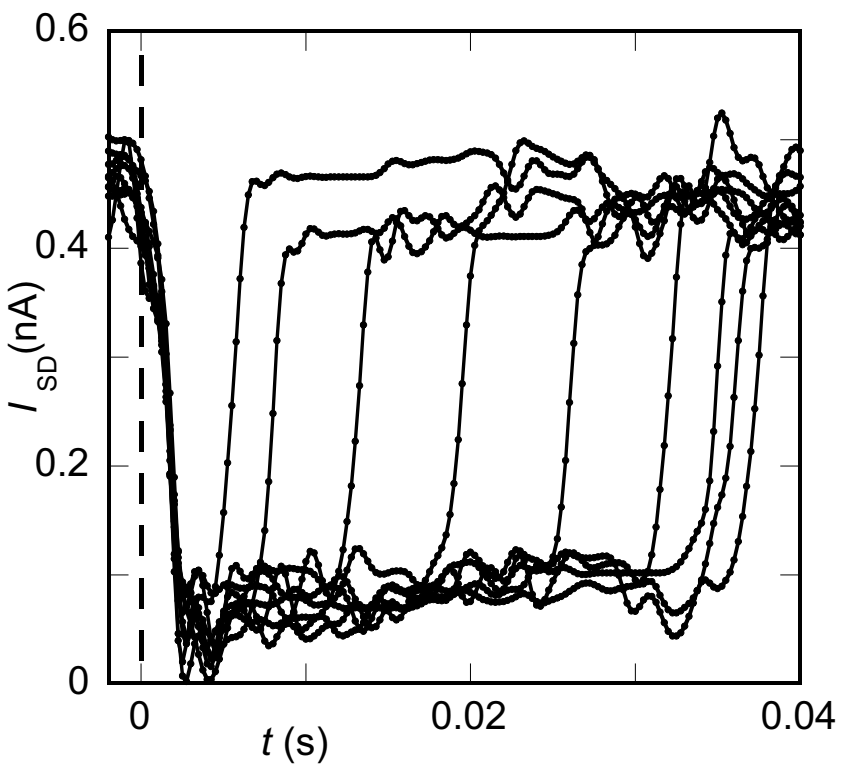


Simkins Figure 11

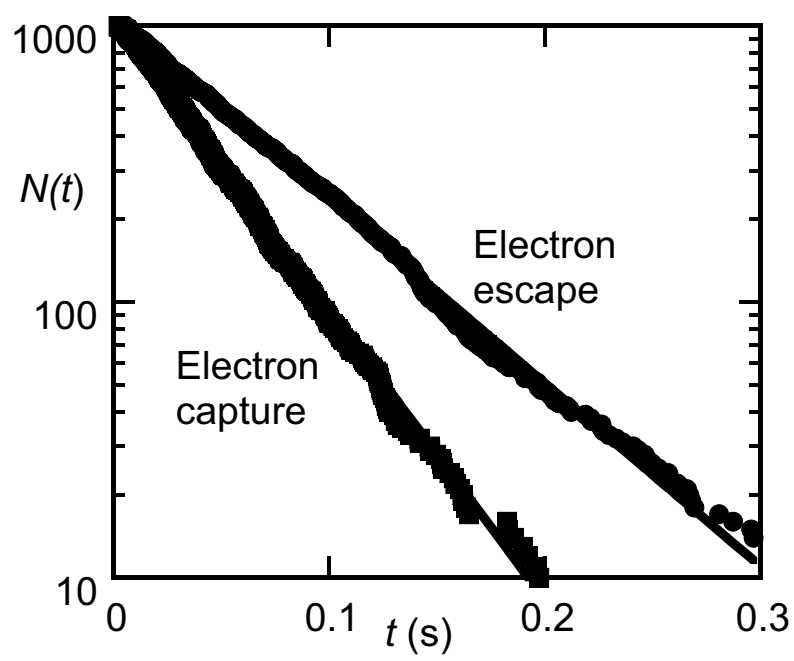


Simkins Figure 12

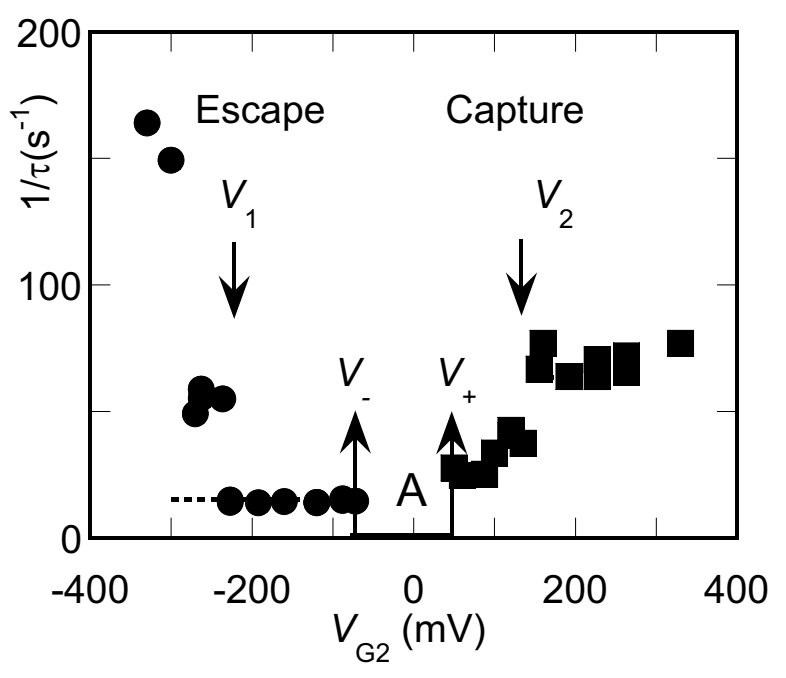



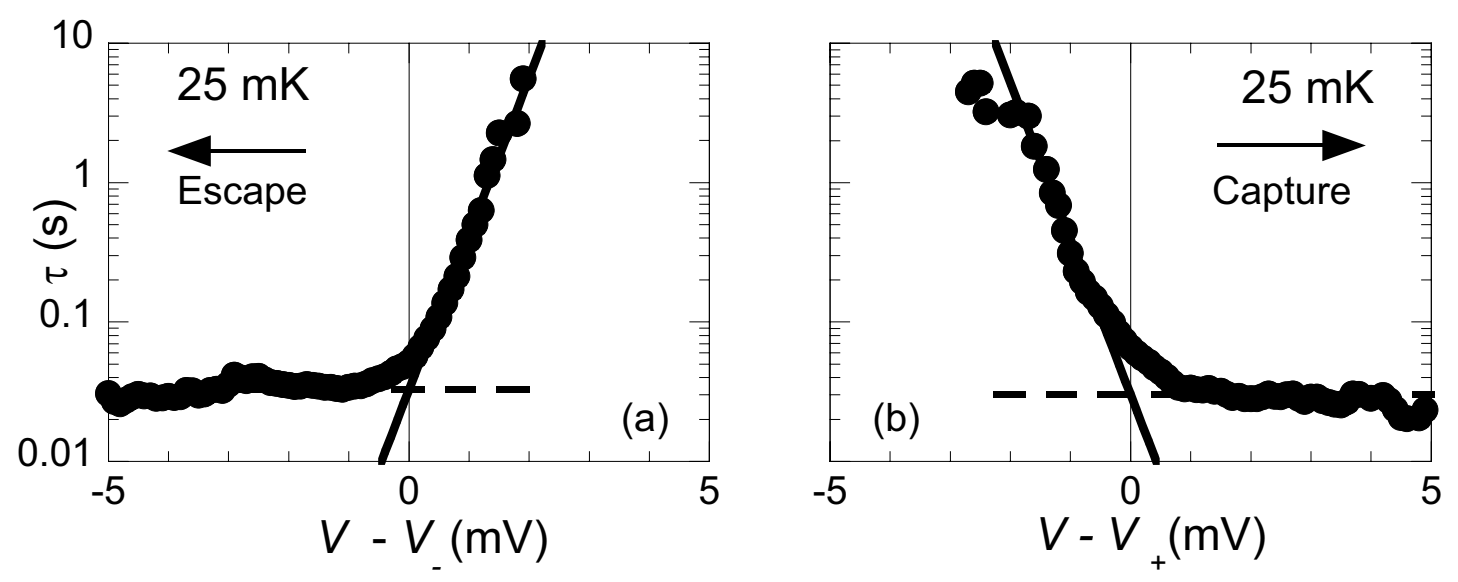

Simkins Figure 13 

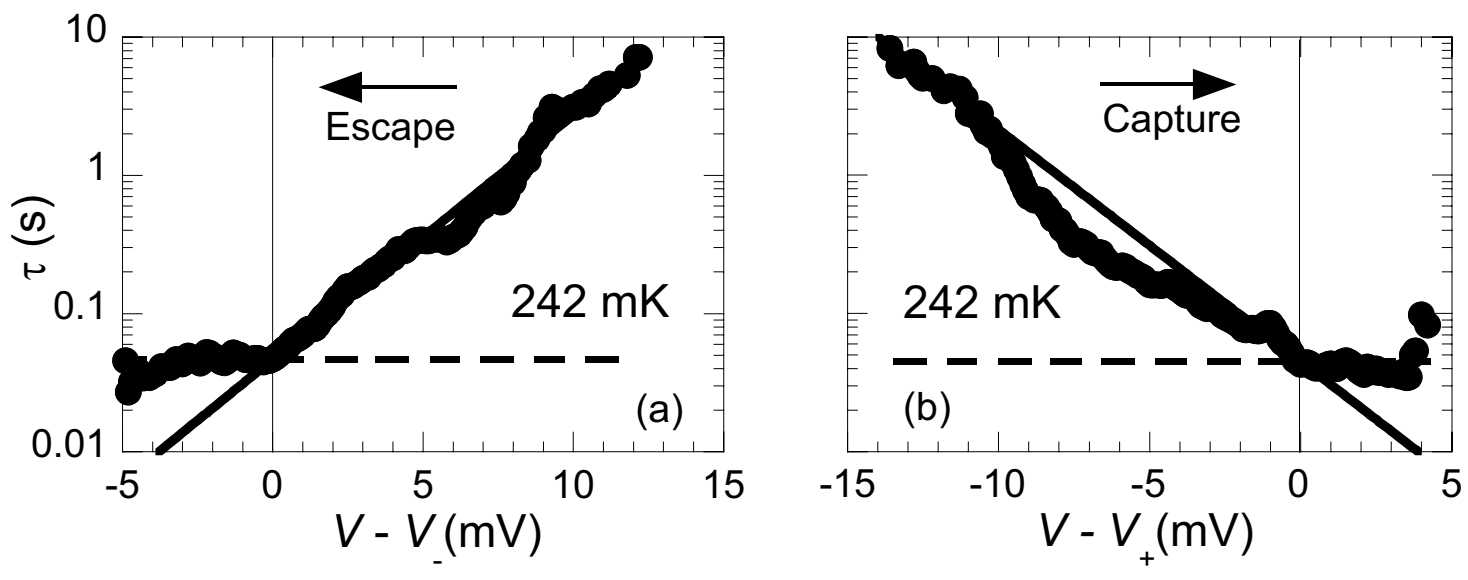

Simkins Figure 14 


\section{Simkins Figure 15}

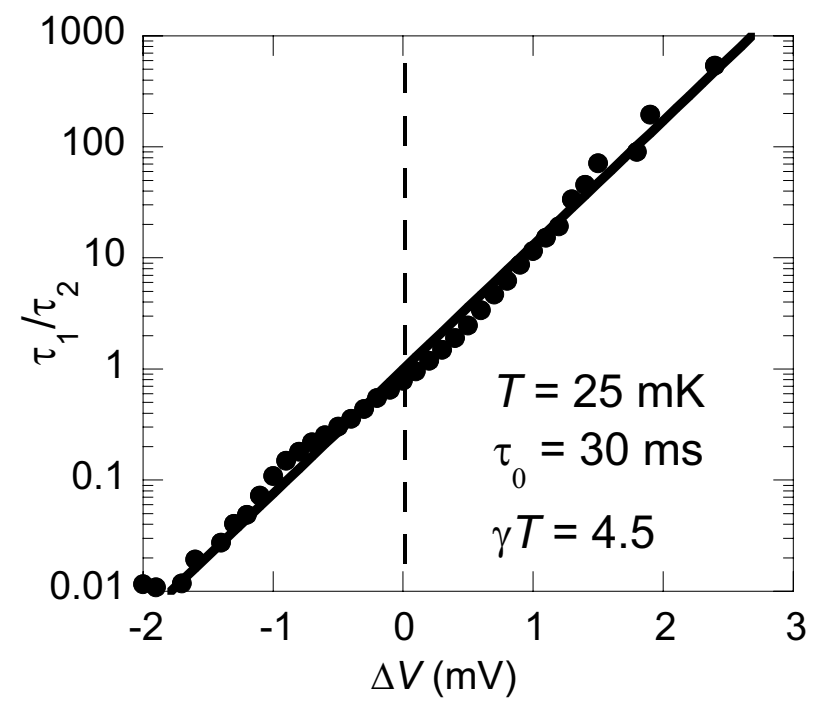



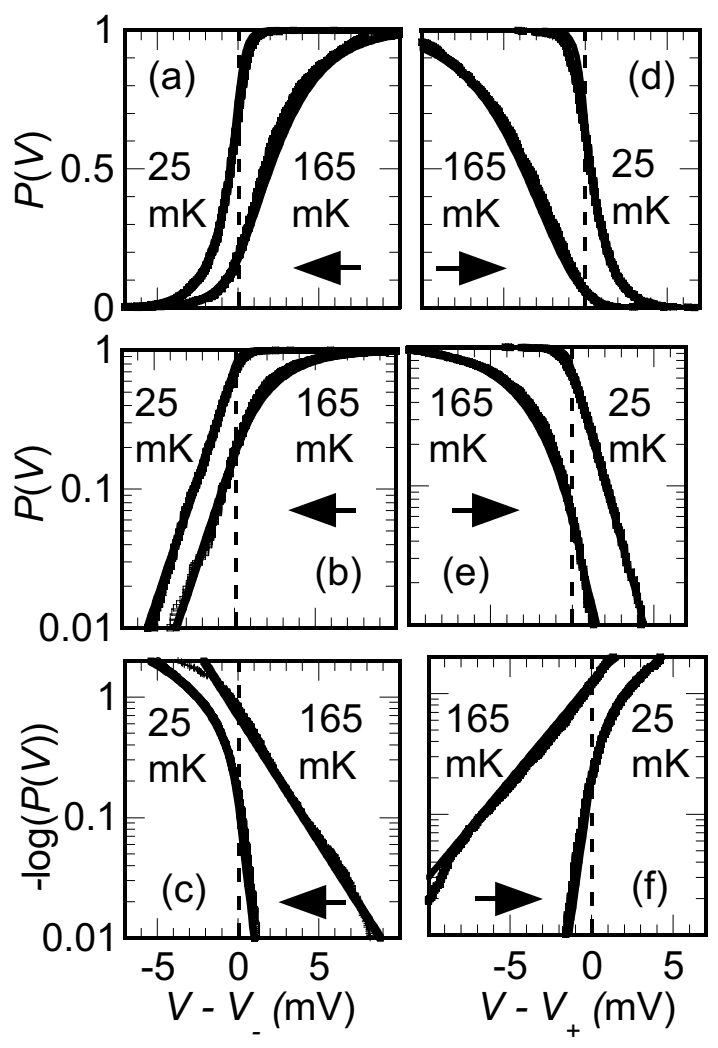

Simkins Figure 16 
Simkins Figure 17

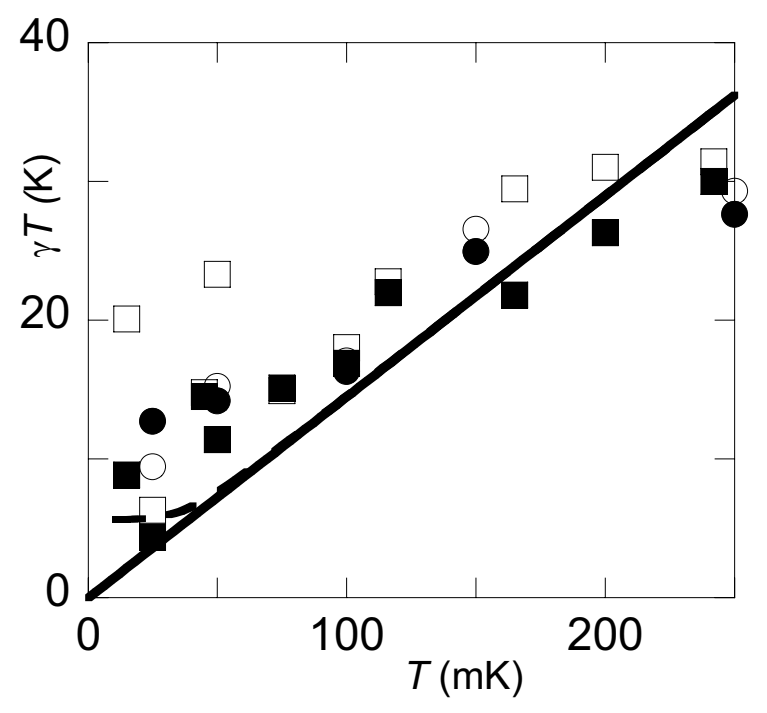




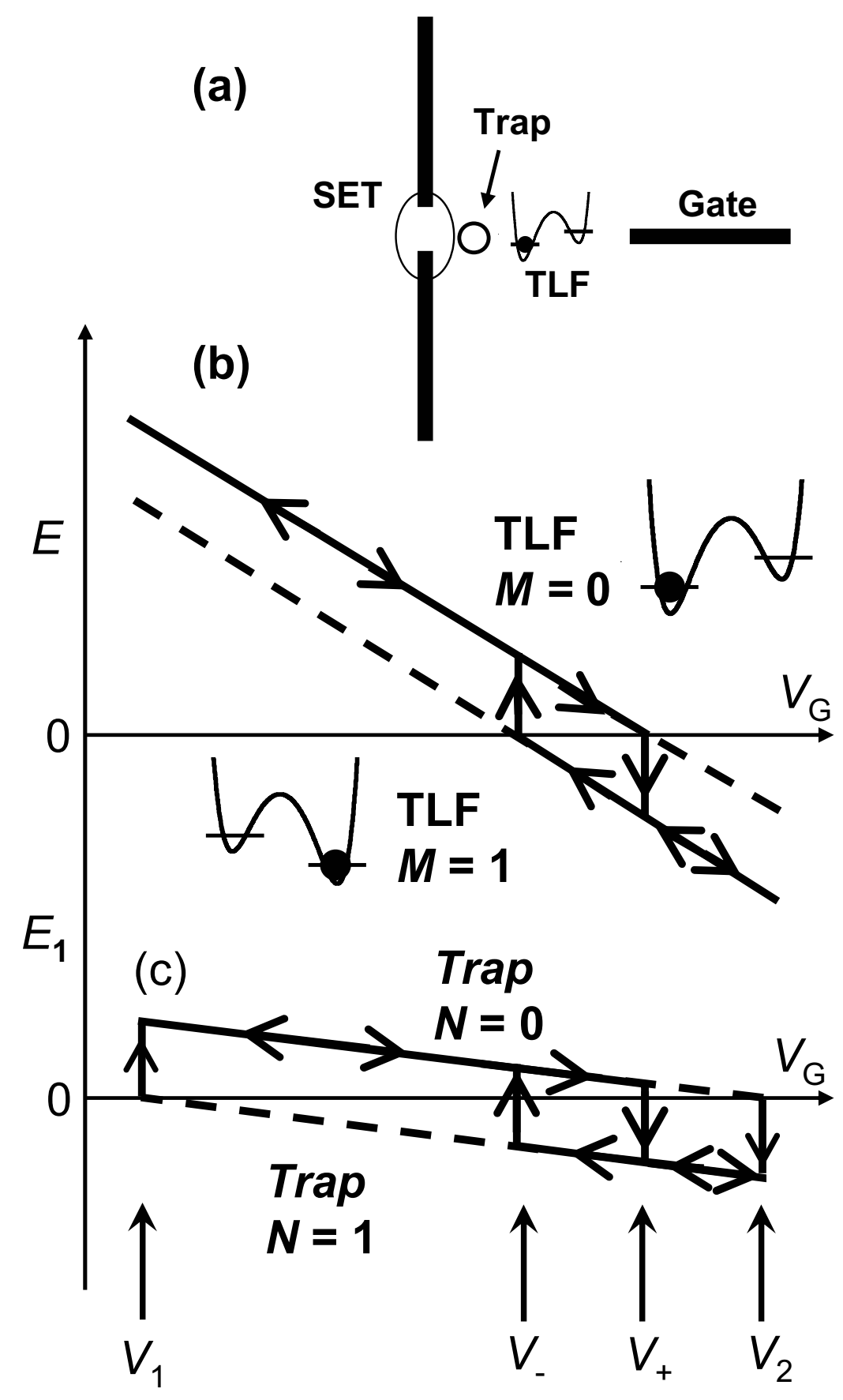

Simkins Figure 18 